\title{
Metastasis: a therapeutic target for cancer
}

\author{
Patricia S Steeg ${ }^{*}$ and \\ Chief of the Women's Cancers Section, Laboratory of Molecular Pharmacology, Center for Cancer \\ Research, National Cancer Institute, Bethesda, MD \\ Dan Theodorescu* \\ Professor of Urologic Oncology and Molecular Physiology and Director of the Paul Mellon Urologic \\ Cancer Institute, University of Virginia, Charlottesville, VA, USA.
}

\section{SUMMARY}

Metastasis remains the major driver of mortality in patients with cancer. Our growing body of knowledge regarding this process provides the basis for the development of molecularly targeted therapeutics aimed at the tumor cell or its interaction with the host microenvironment. Here we discuss the similarity and differences between primary tumors and metastases, pathways controlling the colonization of a distant organ, and incorporation of antimetastatic therapies into clinical testing.

\section{Keywords}

angiogenesis; bone; colonization; metastasis; tumorigenesis

\section{INTRODUCTION}

Despite years of therapeutic development and testing, mortality has improved only incrementally - a few months at best, for many cancer types. Most patients with cancer succumb to metastatic disease or the complications of its treatment. These facts suggest the hypothesis that a mechanistic understanding of the metastatic process and the development of antimetastatic therapies may provide additional reductions in patient morbidity and mortality.

The metastatic process involves tumor cell invasion from the primary tumor, intra vasation, arrest and extravasation of the circulatory system, followed by angiogenesis and growth at a distant site. ${ }^{1,2}$ Metastatic progression is measured by the number and size of large lesions on imaging and by indices of patient survival.

Most of our data in cancer pertains to tumorigenesis. A minority of metastatic lesions is resected, so most histopathologic studies investigate primary tumor tissues. While many tumor cell lines were established from metastatic lesions, most fail to retain an in vivo metastatic phenotype upon prolonged culture. Furthermore, most drug development focuses on short-term changes in primary tumor size in preclinical rodent experiments. Is this a mistake? Is there evidence that the molecular wiring of a metastatic lesion is distinct from our commonly used tumorigenic lines and tissues?

(C2008 Nature Publishing Group

*Correspondence Laboratory of Molecular Pharmacology, National Cancer Institute, Room 1122, Building 37, 37 Convent Drive, Bethesda, MD 20892, USA, E-mail: steegp@ mail.nih.gov. University of Virginia, Room 2561, 2nd Floor, West Clinics, Hospital Drive, Charlottesville, VA 22908-0422, USA, E-mail: theodorescu@virginia.edu.

Competing interests

The authors declared no competing interests. 


\section{PRIMARY TUMORS AND METASTASES: SEPARATE AND UNEQUAL}

\section{Expression trends}

Figure 1 illustrates trends for colorectal carcinoma, for which the resection of liver metastases is occasionally performed. Supplementary Table 1 shows a more complete listing of studies regarding matched primary and metastatic lesions, in addition to a meta-analysis of multiple comparative genomic hybridization (CGH) studies. The expression of genes from matched sets of primary tumors and meta stases co-clustered in profiling experiments, indicating their overall similarity. The data do not, however, indicate their complete identity. Studies have identified distinct expression trends at the RNA or protein levels in primary tumors and metastases, including genes that control meta stasis (MTA1, N-Wasp, NCAML1), extra cellular matrix function (fibronectin, collagens), microtubule dynamics (stathmin), transcription (Snail), drug-processing enzymes (DPD, TS) and kinases (Yes1). ${ }^{3-9}$ Differences occurred both homogeneously and heterogeneously in the matched sets examined. ${ }^{10}$

The quantification issues related to comparisons of matched primary tumors and metastases assessed using CGH, fluorescence in situ hybridization (FISH), and mutation analyses are more straightforward. A meta-analysis showed that the development of liver metastases in patients with colorectal cancer was accompanied by a series of chromosomal deletions and gains in at least $15 \%$ of the tumor specimens; this finding raises the issue of heterogeneity. ${ }^{11}$ Lung meta stases showed more genomic alterations than liver metastases. Analyses of primary tumormetastasis colorectal carcinoma sets showed that the Ki-ras mutational status was discordant in $30 \%, 12$ and FISH analysis demonstrated that $27 \%$ of lung cancer primary tumors and meta stases were discordant in EGFR copy number.

The variability between metastases within a single patient and between patients could be answered by rapid autopsy. ${ }^{13}$ In summary, the expression profiles produced for primary tumors and matched metastases are generally concordant; however, differences in expression do exist, and prompt two further questions: First, what genes or pathways are mechanistically involved in distinguishing primary tumors and meta stases? Second, do these distinctions make a difference - that is, do primary tumors and metastases respond differently to therapeutics?

\section{Mechanistic pathways that distinguish metastases}

Many of the molecular pathways that promote tumorigenesis also promote metastasis and are important in the treatment of both aspects of cancer progression. Some genes exert effects only on metastatic capability, and many of these are relevant to metastatic colonization. Metastasis suppressor genes represent prime examples of metastasis-specific regulation. ${ }^{14}$ Most meta stasis suppressors were identified on the basis of their reduced expression in highly metastatic versus poorly metastatic cell lines or tissues. Transfection of a metastasis suppressor gene into a metastatic cell line resulted in decreased metastatic capability with no significant effect on primary tumor size. Most metastasis suppressors inhibit late steps in the metastatic cascade: tumor cells expressing the Kiss 1 and MKK4 metastasis suppressors arrive in the lungs at frequencies comparable to control transfectants but fail to form large metastases. ${ }^{15,16}$ Specific signaling pathways affected by metastasis suppressors in colonization include Nm23 modulation of the Erk pathway, Brms1 alteration of phosphoinositide signaling, and Mkk4 activation of Jnk and $\mathrm{p} 38$ stress pathways. ${ }^{14}$ Some genes promote metastasis without affecting tumorigenesis, ${ }^{17,18}$ while others affect the host interaction with the metastasizing tumor cell, such as Src-regulated vascular permeability. ${ }^{19}$

\section{Primary tumors and metastases: sensitivity to therapeutics}

Primary tumors and metastases exhibit minor but important differences; however, this is a merely academic argument if they always respond similarly to environmental conditions or 
drugs. Table 1 summarizes results generated in model systems, in which various treatments have produced disparate effects on primary tumor growth and metastases. These studies include diverse cancer histologies and compounds that target multiple pathways. In summary, primary tumors and metastases can differ in expression profiles. They can use distinct molecular pathways and drugs can differentially affect their development. Given these possibilities, we advocate the incorporation of meta stasis models in drug-development studies.

\section{METASTATIC COLONIZATION}

It is important to understand that, in the metastatic process, not all the component processes may be of comparable therapeutic benefit. We propose that the final steps in the metastatic process, outgrowth at a distant site (herein termed 'metastatic colonization'), may hold the most therapeutic promise. Molecular assays will be used to reliably segregate patients with cancer into three categories within the foreseeable future: those at very low risk of metastasis, for whom no further treatment can be considered; those at high risk, in whom distant metastases have not been detected; and those with known metastatic disease. The high-risk group might benefit most from therapies that interrupt metastatic colonization. National Cancer Institute Surveillance Epidemiology and End Result (SEER) data indicate that more than 20\% of patients with breast, kidney and pancreas cancers; more than $30 \%$ of patients with colon, cervix, lung and stomach cancers; and more than $40 \%$ of patients with oral cancers comprise this category at the time of initial diagnosis and surgery. ${ }^{1}$ At this stage of disease, tumor samples will show evidence of invasion, meaning it is too late to stop this aspect of metastasis from occurring. It is the growth of distant micro metastases to clinically detectable, large, lifethreatening metastases (metastatic colonization) that remains incomplete in this setting and, thus, vulnerable to therapeutic intervention.

Metastatic colonization involves reciprocal interactions between tumor cells and a foreign microenvironment. Microenvironments consist of extracellular matrix and normal cells such as fibroblasts, endothelial cells and infiltrating inflammatory cells. Products of these resident and transient cells include growth factors, chemokines, cytokines and proteases.

Microenvironments that contain tumor cells are distinct from normal tissues. Differences include the presence of hypoxia that drives angiogenesis and invasion, low $\mathrm{pH}$, low glucose concentrations, alterations in extracellular matrix proteins and liberation of previously bound growth factors. 20,21

Figure 2 summarizes steps involved in metastatic colonization. Some colonization can occur in the vasculature of the distant organ. ${ }^{22}$ Most cells, however, extravasate and then begin colonization within the organ parenchyma. Hematopoietic precursor cells can migrate from the bone marrow to potential sites of metastasis, which they then condition as a 'premetastatic niche' upon which tumor cells then metastasize. ${ }^{23}$ Tumor cell growth in a foreign microenvironment may be distinct from that at the primary tumor site, selecting for either autonomous growth or survival and proliferation in response to local or circulatory signals. An excellent example is the context-specific activation of the meta stasis suppressors MKK4 and MKK7 in prostate tumor cells disseminated to the lung, but not in the primary tumor. ${ }^{24}$ Angiogenesis or the use (co-option) of existing vessels is required for metastatic colonization beyond the limits of oxygen diffusion and also to provide growth factors, metabolites and nutrients. Metastatic colonization might not be a continuous process. Metastatic tumor cells can enter periods of dormancy at any stage of colonization. Both general and tissue-specific pathways influence metastatic colonization.

\section{Angiogenesis and vascular permeability}

The relationship between vascularity and metastatic colonization is complex. Hypoxic conditions in tumors stimulate angiogenesis. Angiogenesis is regulated by the balance of 
angiogenic and antiangiogenic factors. The most widely studied angiogenic factor is VEGF, which induces the proliferation, migration and organization of endothelial cells to form new capillaries. VEGF has several isoforms and is a multi functional protein. In addition to its role in angio genesis, VEGF regulates vascular permeability, the proliferation of some tumor cells and the formation of a premeta static niche. ${ }^{1}$ Overexpression of $V E G F$ induced greater numbers of tumor micro vessels and increased metastasis. ${ }^{25}$ Both the angiogenic and vascular permeability functions can contribute to VEGF regulation of metastatic coloni zation. ${ }^{19}$ Bevacizumab, a humanized monoclonal antibody to VEGF, has clinical activity in combination with cytotoxics in multiple cancer histologies. ${ }^{26,27}$

A gene-expression analysis of primary breast tumors and metastasis samples identified complex differential expression patterns of multiple angiogenic and antiangiogenic factors between tumor sites and between patients. ${ }^{28}$ The sprouting of capillaries is not necessarily the ultimate end point of angiogenesis, and signaling involved in pericyte coverage and barrier function may be thera peutically important. In a prostate cancer model system, low levels of the CXCL12 chemokine were found in the primary tumor, which permitted the CXCR4 chemokine receptor to stimulate tumor secretion of the glycolytic enzyme phospho glycerate kinase 1 (PGK1). PGK1 then induced the secretion of the antiangiogenic factor angiostatin and inhibited production of VEGF and inter leukins 6 and 8 (IL-6, IL-8). In metastatic sites such as the bone and liver, however, ample CXCL12 negated this pathway, resulting in less PGK1 and angiostatin and greater angiogenesis factor production, facilitating angiogenesis and coloni zation. ${ }^{31}$ In preclinical models, combinations of anti angiogenesis tactics showed optimum efficacy. 29,30

Experimental evidence supports several alternative scenarios to the traditional angiogenesis concept. First, both liver and brain metastatic tumor cells can co-opt the existing host vasculature rather than induce angiogenesis. ${ }^{32,33}$ In the brain, co-option of the existing vasculature resulted in an intact blood-brain barrier, which could contribute to chemotherapy resistance even in large metastases. Second, recent reports correlated increased vascular density directly with poor tumor growth. This apparent contradiction was explained by the contri bution of Dll4, which stimulated angiogenesis to a nonproductive level, resulting in poor perfusion, increased hypoxia and reduced tumor outgrowth. ${ }^{34,35}$ Dll4 blockade was demonstrated to reduce the growth of VEGF-inhibitor-resistant experimental tumors.

\section{Metastatic dormancy}

Tumor dormancy is a well-known clinical phenomenon. Metastases from certain histo logical types of cancer can occur more than 10 years after successful treatment for the primary tumor. Fatal melanoma was noted in patients who underwent kidney transplant 16 years after surgical cure. ${ }^{36}$ Dormant cells are plastic, highly regulated by their interaction with the metastatic micro environment. Dormant tumor cells have been harvested from mouse tissues, expanded in tissue culture, and re injected into animals to produce growing primary tumors that spawned dormant micro metastases. ${ }^{37}$ Experimental data support two models of dormancy in the metastatic site: first, tumor cells that lack a vascular connection and balance apoptosis and proliferation; 38 and, second, tumor cells that enter a viable but non dividing phase.

Several molecular pathways operative in metastatic dormancy have been identified in model systems. The myc, integrin, Bcl-Xl anti apoptotic and p38 stress pathways have been shown to influence tumor dormancy. KISS1, the first secreted metastasis suppressor, induced and maintained the dormancy of melanoma cells. ${ }^{15}$ Another postulated antidormancy strategy involves thrombin, which can impact proliferation through the protease-activated receptor. 39 


\section{Drug responsiveness}

Metastatic colonization might affect drug responsiveness, and vice versa. Tumor cells that grow in the bone-marrow microenvironment stimulate bone marrow production of 'survival' factors such as IL-6 and insulin-like growth factors (IGFs), which protect the tumor cells from cytotoxic drugs. ${ }^{40}$ Similarly, bone marrow cells produce osteoprotegerin (OPG) that protects bone metastatic breast-cancer cells from apoptosis induced by TRAIL. ${ }^{41}$ Conversely, drugs can modify the interaction between tumor cell and micro environment, which can impact metastatic colonization. Analysis of biopsy samples from a clinical trial of pre operative thalidomide in patients with prostate cancer revealed changes in protein expression in the metastatic microenvironment (including tumor cells, endothelial cells and stromal cells) that favored a less metastatic state. ${ }^{42}$ Further mechanistic advances in this research area are vital to therapeutic advances.

\section{Site-specific colonization to the bone}

This Review will focus on bone metastasis as an example of progress and challenges in sitespecific metastatic colonization. The delineation of bone metastatic signaling pathways was fostered by the development of in vivo metastasis assays. These model systems have limitations and require validation to confirm that the model is actually representative of molecular events found in the human disease. In addition, a single model cannot represent the heterogeneity found in human disease. ${ }^{1,43-45}$

Normal bone remodeling is a dynamic balance of osteoblastic (bone forming) and osteolytic (bone degrading) activity. Breast and prostate cancers and multiple myeloma most frequently produce bone metastases. A pathologic analysis of resected breast cancer osteoclastic lesions showed that $72 \%$ of the lesions were a mixture of osteoclastic and osteoblastic, while the remaining $28 \%$ were purely osteolytic. Osteolytic metastases can evolve through a series of tumor-microenvironment interactions known as the 'vicious cycle' (Figure 3). ${ }^{46}$ Tumor cells secrete factors that stimulate osteoblast activation of osteoclasts. Activated osteoclasts degrade the bone matrix and release factors that stimulate tumor cells. Many other proteins also participate in osteolytic bone metastasis. ${ }^{1,46}$ Overexpression of the chemokine CXCR4 increased bone metastasis in a model system and this activity was enhanced by coordinate expression of IL-11, osteopontin and/or connective tissue growth factor. ${ }^{47}$

An autopsy study of bone metastases from androgen-independent prostate cancer found tumor cells at an average of 14 sites within the marrow. Heterogeneity was observed between metastases from a single patient in the fraction of tumor cells that were positive for prostatespecific antigen (PSA) or chromogranin A (CGA) ${ }^{48}$ No single vicious cycle has been mapped out for osteoblastic lesions. Debate continues on the relative and temporal role of osteolysis. Osteoblastic activity may be increased by PSA cleavage of parathyroid-hormone-related protein, breaking the osteolytic vicious cycle. ${ }^{46}$ In addition, growth factors such as transforming growth factor- $\beta$ (TGF- $\beta$ ), fibroblast growth factor (FGF), IGF and endothelin-1 (ET-1) activate the RUNX2 transcription factor in osteoblasts, which results in osteoblastic gene expression. 49

Numerous compounds have been tested in model systems for potential activity against bone metastases (Supplementary Table 2). These include compounds that are active against confirmed bone metastasis targets (OPG, TGF- $\beta$, and colony-stimulating factor), in addition to others that target tumor cells more generally. Most model systems involve injection of tumor cells into the circulation; alternatively, tumor cells can be directly implanted into bone, which elimi nates the extravasation and initial micro metastatic colonization events. Few studies permit a meta stasis to grow before asking whether the compound will have an antitumor effect, comparable to early clinical trials conducted in the metastatic setting. Even fewer studies report 
pharmacokinetic data. One reason why so many mice, but not humans, are cured may be that mice are given compounds at levels unachievable in humans.

\section{CLINICAL TARGETING OF METASTATIC COLONIZATION}

Most anticancer agents move through a defined series of clinical tests, beginning with phase I dosefinding trials in the relapsed metastatic setting. Progressively, agents move to efficacy testing in phase II trials and a head-to-head comparison with the standard of care in phase III trials. These trials measure the effect of a drug on the dissolution of established metastatic disease as well as the prevention of new overt metastases. If metastatic colonization is an important therapeutic target, how should it be clinically validated? Are current paradigms adequate? What patient cohorts should be selected? Another unanswered question is whether compounds active in halting metastatic colonization will be effective in the setting of established metastatic disease. Parthenolide, a NF- $\kappa B$ pathway inhibitor, was ineffective in a therapeutic preclinical model of osteosarcoma metastasis, but prevented metastatic colonization in a prophylactic model. ${ }^{50}$ By contrast, an RGD peptidomimetic agent inhibited the lung metastasis of breast cancer cells when administered either at the time of tumor cell injection or after visible pulmonary metastases had appeared. 51

HER 2 overexpression occurs in approximately $25 \%$ of breast cancers and contributes to both tumor growth and metastasis. In a preclinical mouse model of bone metastasis, the monoclonal antibody trastuzumab prevented the outgrowth of bone lesions in mice injected with breast carcinoma cells overexpressing $H E R 2$; if trastuzumab therapy was delayed until bone lesions were evident, the drug only maintained the size of the extant lesions. ${ }^{52}$ Similar trends were apparent in clinical testing: trastuzumab in combination with cytotoxic chemotherapy was effective in the metastatic setting, increasing the objective response rate by $18 \%$ and median survival by 4.8 months. ${ }^{53}$ In the adjuvant setting, where a similar drug combination prevented the outgrowth of occult disseminated disease in patients with no detectable distant metastatic disease, progression was reduced to nearly half that of the control group over a short followup period. ${ }^{54,55}$ These data suggest that agents with activity in metastatic colonization will show optimum activity in the adjuvant setting.

\section{Box 1}

Potential elements that support the conduct of clinical trials of drugs targeting metastatic colonization in the adjuvant setting.

The effect of a drug on metastatic colonization may be best measured in the adjuvant clinical setting. In the adjuvant setting, the drug should be administered to patients who are without detectable distant metastatic disease, but are at high risk for its development-for instance, breast cancer patients with tumor cells in the axillary lymph nodes. Given the expense, large size and time required for adjuvant trials, what data should optimally be available? The following are suggested:

- Did the compound impact the molecular target in multiple preclinical models? In the clinic?

- Did the compound elicit stable disease (>6 months) or, less likely, objective clinical responses in the metastatic setting?

- Is the drug active at all sites or is it site-specific?

- Has an optimum rational combination been identified and tested clinically in the metastatic setting? 
- Have the patient selection criteria been optimally chosen for the molecular target or metastatic tissue?

- What was the duration of treatment?

- Are the end points adequately delineated?

- Are validated biomarkers of response being used?

\section{Clinical trial design considerations}

Depending on the cancer histology and trial design, adjuvant trials can be large, expensive and/ or slow to complete. The 'bar' for the conduct of these studies is necessarily high. Box 1 lists some potential components that support the conduct of adjuvant trials for therapeutics directed at metastasis. While high rates of objective responses on established lesions may not be amassed in early testing, stable disease-usually defined over a 6-month period-is expected in the metastatic setting. Standardization of the stable disease metric is needed.

Another critical question is whether the drug acts on the intended molecular target. Studies in mice that address this question are easy to perform because tissues are readily available; however, data from murine studies might not apply directly to humans. Study designs in humans include the acquisition of pretreatment and post-treatment biopsies of metastatic lesions for pharmacodynamic experiments. Consent for multiple biopsies can be difficult, the specimens may contain unusable necrotic tissue, and the procedures are often not covered by insurance reimbursement. Another design is a preoperative therapy trial, in which the tumor is biopsied and the patient then undergoes chemotherapy before full surgical excision. The pretreatment biopsy is compared with the treated excised tumor for pharmacodynamic measurements. Other approaches include using peripheral blood cells or other surrogate tissues as a biomarker of drug response, which might reflect the physiology of a metastatic lesion. For instance, early clinical testing of potential bone metastasis inhibitors used markers of bone formation or destruction in serum or urine. ${ }^{56-58}$

Another factor is the selection of patients who are at high risk of disease recurrence. Depending on the cancer histology, 'prognostic' tools that use conventional information, possibly combined with molecular profiling, are being developed to address this issue. For example, in bladder cancer, radical cystectomy is a standard treatment for localized and regionally advanced invasive disease. An international bladder-cancer database was constructed from patients treated in this way and a nomogram to predict the 5-year disease recurrence risk was developed. ${ }^{59}$ Few gene-expression profiles, however, have been prospectively validated.

Will antiangiogenic therapy enjoy success in the adjuvant setting similar to that which was observed for anti-HER2 therapies? Bevacizumab has shown limited clinical activity in the metastatic setting in combi nation with cytotoxic drugs (Supplementary Table 3). The degree of patient pre treatment was an important factor in patient out-come. In certain trials, responses or changes in time to progression were observed in the absence of an effect on survival, making it difficult to interpret any clinical benefit. With reference to the 'wish list' in Box 1, the mechanism of action of bevacizumab seems complex. In a preoperative study of inflammatory or locally advanced breast cancer, bevacizumab decreased phosphorylated VEGFR2, increased tumor-cell apoptosis and altered flow parameters on dynamic-contrast enhanced MRI, but failed to reduce tumor microvessel density or circulating VEGFA concentrations. ${ }^{60}$ This observation was confirmed and extended in a colorectal cancer trial: tumor microvessel density and serum VEGF levels and thrombospondin were not predictive of bevacizumab activity. ${ }^{61}$ A Breast Cancer Intergroup adjuvant trial, E5103, will examine the addition of bevacizumab 
to standard chemotherapy in high-risk lymph-node-negative and lymph-node-positive patients (GW Sledge, personal communication).

Clinical testing in patients with resectable metastatic disease may be ideal for drugs that affect metastatic colonization. Colorectal and renal cell carcinomas represent two well-studied examples. One such clinical example involves patients with limited metastases from colorectal cancer to the liver and lungs. These patients may benefit from resection of their metastatic sites, with cure rates approaching 50\%; however, $50 \%$ or more will experience recurrence from pre-established microscopic disease. ${ }^{62}$ Such patients are ideal candidates for trials in which random assignment to an adjuvant post resection versus a placebo postresection would allow determination of the adjuvant's ability to improve disease-free survival and time to recurrence. Such a trial has been conducted for thalido mide (SK Libutti, personal communication). Alternatively, patients could have a sentinel or dominant metastatic lesion biopsied and then be randomized to undergo a short neoadjuvant therapy with the investi gational agent before surgical resection. The tumor would then be resected and a molecular and pathologic analysis of the tissues taken before and after treatment would determine biomarkers of response as well as evidence of a biological effect on the target. ${ }^{63}$ If the pharmacodynamic and pathologic response was favorable, the patients would be continued on the drug in an adjuvant setting.

\section{Rational combinations might be most effective}

Simplistically, it is assumed that combinations of drugs that target metastatic colonization will give greater benefit than single agents. This supposition is in agreement with the efficacy of poly chemotherapy as opposed to monotherapy. While single genes are described as 'dominant' or 'addictive' in primary tumor mouse models, it is questionable whether these terms are still meaningful in the context of genomically and phenotypically unstable metastatic disease. For most clinical trials, complete responses are rare and de novo or acquired resistance to single molecularly targeted agents is prominent. Potential drug combinations for metastatic colonization can involve diverse aspects of the tumor cells or their micro environment. There are more rational combinations of agents that could be used against metastatic colonization than there are the clinical trial patients, time and resources to test them.

Angiogenesis is a prime example of a potential target for combinations of antimetastatic therapeutics (Figure 4). An antiangiogenic and targeted therapy combination that underwent preclinical and clinical investigation is bevacizumab with EGFR tyrosine kinase inhibitors. A simple model postulates that this strategy would target both endothelial cells and tumor cells. The potential synergy is complex, because both endothelial and tumor cells express EGFR and $V E G F R$. Further, stimulation of tumor cells with EGF induced VEGF production. Multiple preclinical reports demonstrated an enhanced response using VEGF/VEGFR and EGFR inhibitory combinations. Of interest is a non randomized phase I/II trial in which patients with stage IIIb/IV non-small-cell lung carcinoma who had undergone one or more cycles of prior chemotherapy were treated with the EGFR inhibitor erlotinib and bevacizumab in the absence of additional cytotoxics. The overall response rate was $20 \%$, with $65 \%$ of patients having stable disease as their best end point. ${ }^{63}$ Similar results were reported for the same combination in patients with clear-cell renal carcinoma ${ }^{64}$ but not in patients with previously treated metastatic breast cancer who received ZD6474 (vandetanib), a tyrosine kinase inhibitor of both VEGFR2 and EGFR. ${ }^{65}$ Further investigation of the drugs, in terms of their pharmacokinetics and mechanisms of action, will be needed to determine the most promising combination.

Multikinase inhibitors such as sorafenib and sunitinib have shown limited activity in metastatic renal carcinoma (Supplementary Table 3). 


\section{Niche trials: the bone metastasis paradigm}

Bone metastasis is an excellent paradigm in which to study site-specific antimetastatic therapies. Bone metastases are debilitating, causing bone pain, fractures and spinal-cord compressions.

The first clinical uses of bisphosphonates (BPs) began almost 40 years ago. In the metastatic setting, clodronate, pamidronate, zoledronic acid and ibandronate have demonstrated efficacy in the treatment of skeletal events. ${ }^{66}$ Preclinical data indicate palliative effects of BPs that are consistent with clinical trial data. Other preclinical models indicated therapeutic effects including the inhibition of tumor cell adhesion to bone, tumor cell growth and viability, and angiogenesis. ${ }^{66}$ Adjuvant trials of BPs in breast cancer have thus far failed to validate a benefit in terms of patient survival. ${ }^{67-70}$ The fact that experimental results have not been reconciled with survival data after 10 years of clinical testing highlights the potential difficulties in trial design and execution of studies examining metastatic colonization. In addition to their use as monotherapy, there are combinations in which BPs might have a significant impact on metastasis. A small trial of 25 patients with hormone-refractory prostate cancer treated with docetaxel and zoledronic acid was reported. ${ }^{71}$ Overall, $48 \%$ of patients had a PSA response and the median time to progression was 7 months.

ABT-627 (atrasentan), an ET-1 receptor antagonist, significantly reduced the osteoblastic response and the number of metastatic lesions in preclinical experiments. ${ }^{72}$ Overall, atrasentan was well tolerated and delayed the progression of hormone-refractory prostate cancer in some men. ${ }^{73}$ One of the trials reported a separate pharmaco dynamic analysis of biological end points that included changes in bone deposition markers such as total alkaline phosphatase, bone alkaline phosphatase and the bone resorption markers $N$-telopeptides, $C$-telopeptides and deoxy pyridinoline. ${ }^{74}$ Analysis revealed that mean changes from baseline in total alkaline phosphatase, bone alkaline phosphatase and deoxy pyridinoline were consistently lower in patients receiving $10 \mathrm{mg}$ atrasentan than in patients receiving placebo.

Molecular therapeutics designed to inhibit bone metastasis have entered clinical testing. A recombinant OPG (AMGN-0007; Figure 3) was tested in a phase I clinical trial in patients with osteolytic bone disease caused by multiple myeloma or breast cancer. ${ }^{75}$ AMGN-0007 caused a rapid, sustained, dose-dependent decrease in urinary $N$-telopeptide and collagen/creatinine levels that was comparable to the profile observed with pamidronate. In addition, the humanized monoclonal antibody denosumab (AMG 162) binds RANKL and inhibits its action. Recently, a study evaluated denosumab in a randomized double-blind setting to determine the safety and efficacy of this antibody in patients with breast cancer or multiple myeloma with confirmed bone meta stasis. 76 Patients received a single dose of either denosumab or pamidronate, and bone anti resorptive effect was assessed by changes in urinary and serum $\mathrm{N}$-telopeptide levels. Interestingly, denosumab treatment resulted in a decrease in levels of urinary and serum $N$ - telopeptide that lasted for 84 days. Pamidronate also decreased bone turnover, but the effect diminished progressively through follow-up.

\section{Novel approaches to metastatic colonization}

Metastasis-related targets other than the traditional tyrosine kinase pathways could have therapeutic potential. Multiple targets have been identified in the literature and validated in meta stasis models. Several targets of particular importance to metastatic colonization such as metastasis suppressors, are discussed.

Three metastasis suppressors illustrate diverse approaches in translating these basic research observations to the clinic. The first approach attempts to elevate metastasis suppressor expression, which was reported for Nm23-H1. High-dose medroxyprogesterone acetate (MPA) 
elevated the Nm23-H1 expression of estrogen-receptor-negative, progesterone receptornegative, glucocorticoid receptor-positive breast cancer cell lines, distinct from stimulatory pathways involving the traditional progesterone receptor. In vivo, MPA reduced the metastatic colonization of hormone receptor-negative, glucocorticoid receptor-positive MDA-MB-231 breast carcinoma cells by 33-62\%. ${ }^{77}$ A phase II trial of high-dose MPA, alone or in combination with low-dose oral cyclophosphamide and methotrexate, for metastatic breast cancer patients with hormone receptor-negative tumors is now open (KD Miller, personal communication).

Microarrays have been used to identify proteins upregulated in cell lines of aggressive disease when the metastasis suppressor was down regulated. A microarray analysis of high-expressing and low-expressing bladder carcinoma cell lines identified ET-1 as a correlate of low RhoGDI2 expression. Atrasentan, an ET-1 receptor antagonist, inhibited the pulmonary metastasis of a low RhoGDI2 expressing bladder cancer cell line by $80 \% .78$ An adjuvant clinical trial using an ET-1 receptor antagonist is currently being planned for patients with locally advanced but resected bladder cancer. A similar approach has identified the lysophosphatidic acid receptor EDG2 as a correlate of low Nm23-H1 expression. ${ }^{79}$ A third approach involves the KISS1 metastasis suppressor. Production of a secreted KISS1 suppressed melanoma metastasis to multiple organs and enhanced median survival almost three-fold. ${ }^{15}$ KISS1 mimetics are under develop ment. 80

A microarray signature for lung-specific metastasis included the EGFR ligand epiregulin, COX2 and the matrix metalloproteinase inhibitors MMP-1 and -2. Administration of a cocktail of inhibitors of these proteins reduced tumor volume and pulmonary metastases. 81

TGF- $\beta$ is an interesting molecule. In normal tissues TGF- $\beta$ inhibits growth by signaling via Smad 2 and Smad3 proteins, which then bind Smad4 and translocate into the nucleus and function in transcriptional regulation. In malignant cells, mutation of this pathway, altered expression of normal or inhibitory Smads and/or cross-interaction with other pathways renders TGF- $\beta$ widely metastasis-inducing. ${ }^{82,83}$ The immunosuppressive activities of TGF- $\beta$ may also contribute to its prometastatic effects. ${ }^{84}$ Standard chemotherapies induce TGF- $\beta$ production, and neutralizing antibodies inhibited this mechanism of metastatic progression. 85 Multiple therapeutic strategies are in development or early clinical testing. ${ }^{86}$ GC-1008 (Genzyme in collaboration with Cambridge Antibody Technology), a human monoclonal $\mathrm{IgG}_{4}$ antibody capable of binding and neutralizing TGF- $\beta$ isoforms 1,2 and 3 , is being evaluated for the treatment of several indications including cancer and diseases associated with fibrosis. Currently, phase I studies to determine the pharmacokinetic properties, dose and safety of GC-1008 are being performed in patients with malignant melanoma and renal cell carcinoma (B Teicher, personal communication). It may be critical to determine a gene-expression signature or other predictive marker of TGF- $\beta$ inhibitory versus stimulatory activities, to identify patients with the greatest potential to benefit from the many TGF- $\beta$-related therapeutic strategies.

The motility receptor c-Met has been linked to the metastatic process. ${ }^{87}$ Multiple attempts to target c-Met or its ligand hepatocyte growth factor (HGF, scatter factor) are underway. ${ }^{88}$ Like c-Met, the nonreceptor tyrosine kinase Src was considered part of an invasion pathway with Fak. Reports now link Src to multiple aspects of metastasis including bone colonization, vascular permeability and angiogenesis. $19,89,90$

Stem cells were reported in metastatic breast cancer biopsies, however, their role in metastatic progression remains controversial. ${ }^{91}$ The pathways controlling the growth and regenerative capacity of stem cells, if validated and distinct from that of common tumor cells, will be of relevance to the continuation of metastatic progression. 
Another drug combination that is likely to be of great interest in terms of metastatic colonization is the use of a cocktail of inhibitors that target receptors and ligands. The preclinical literature is replete with studies indicating that resistance to the inhibition of a growth factor receptor is linked to activation or crosstalk from another receptor. Candidates of great interest include the IGF-1R, c-Met, and the EDG receptors for lysophosphatidic acid. Many of these inhibitors are still in preclinical testing or early clinical trials as single agents. Key to the success of this effort will be the conduct of trials using drug combinations produced by multiple companies.

\section{CONCLUSIONS}

It is time for agents that target metastasis and, in particular, metastatic colonization, to enter mainstream clinical development and testing. We hypothesize that the addition of these targets to the current armamentarium will ultimately enhance the efficacy of current treatment regimens. There is a lot of work remaining, including the selection of optimum drug combinations and the optimization of multiple aspects of trial design. In addition, we must garner the willpower to conduct adjuvant trials when fantastic objective response rates are not seen in the metastatic setting-it can be done.

KEY POINTS
Most information on human cancer is obtained from analysis of primary tumors
and yet this knowledge is applied to the treatment of metastases
There is mounting genetic evidence that the molecular wiring of a metastatic lesion
has both elements in common with and elements that are distinct from those of
primary tumors
Targeting the last step in the metastatic process, outgrowth at a distant site, termed
'metastatic colonization', holds great therapeutic promise
Blockade of metastatic colonization can be accomplished by targeting the
metastatic cancer cell or the host cell, or by interrupting reciprocal interactions
between tumor cells and the foreign microenvironment; therapeutic efforts can
target metastatic colonization at all sites or interactions specific to a particular
organ (bone, for instance)
Novel clinical trial designs with short-term molecular and pharmacodynamic end
points should be considered
Approaches to inhibit metastatic colonization may show their best efficacy in the
adjuvant setting

\section{REVIEW CRITERIA}

The information for this Review was compiled by searching the PubMed and Institute for Scientific Information (ISI) databases for articles published up to 14 August 2007. Electronic early-release publications were also included. Only articles published in English were considered. The search terms used included "metastasis" in association with other search terms: "primary tumor", "drug", "angiogenesis", "preclinical”, "bevacizumab", "trastuzumab", "microenvironment". When possible, primary sources have been cited. The results of some experiments and trials conveyed to the authors by personal communication were also included. References were chosen on the basis of the best clinical or laboratory evidence, especially if the work had been corroborated by published work from other centers. 


\section{Supplementary Material}

Refer to Web version on PubMed Central for supplementary material.

\section{References}

1. Steeg PS. Tumor metastasis: mechanistic insights and clinical challenges. Nat Med 2006;12:895-904. [PubMed: 16892035]

2. Gupta GP, Massague J. Cancer metastasis: building a framework. Cell 2006;127:679-695. [PubMed: 17110329]

3. Hoang CD, et al. Analysis of paired primary lung and lymph node tumor cells: a model of metastatic potential by multiple genetic programs. Cancer Detect Prev 2005;29:509-517. [PubMed: 16289389]

4. Roepman P, et al. Maintenance of head and neck tumor gene expression profiles upon lymph node metastasis. Cancer Res 2006;66:11110-11114. [PubMed: 17145852]

5. Wang L, et al. Comparison of gene expression profiles between primary tumor and metastatic lesions in gastric cancer patients using laser microdissection and cDNA microarray. World J Gastroenterol 2006;12:6949-6954. [PubMed: 17109515]

6. Yanagawa R, et al. Genome-wide screening of genes showing altered expression in liver metastases of human colorectal cancers by cDNA microarray. Neoplasia 2001;3:395-401. [PubMed: 11687950]

7. Hao X, et al. Differential gene and protein expression in primary breast malignancies and their lymph node metastases as revealed by combined cDNA microarray and tissue microarray analysis. Cancer 2004;100:1110-1122. [PubMed: 15022276]

8. Inokuchi M, et al. Gene expression of 5-fluorouracil metabolic enzymes in primary colorectal cancer and corresponding liver metastasis. Cancer Chemother Pharmacol 2004;53:391-396. [PubMed: 15060742]

9. Feldmann G, et al. Blockade of hedgehog signaling inhibits pancreatic cancer invasion and metastases: a new paradigm for combination therapy in solid cancers. Cancer Res 2007;67:2187-2196. [PubMed: 17332349]

10. Suzuki M, Tarin D. Gene expression profiling of human lymph node metastases and matched primary breast carcinomas: clinical implications. Mol Oncol 2007;1:172-180. [PubMed: 19383293]

11. Diep CB, et al. The order of genetic events associated with colorectal cancer progression inferred from meta-analysis of copy number changes. Genes Chromosomes Cancer 2006;45:31-41. [PubMed: 16145679]

12. Albanese I, et al. Heterogeneity within and between primary colorectal carcinomas and matched metastases as revealed by analysis of Ki-ras and p53 mutations. Biochem Biophys Res Commun 2004;325:784-791. [PubMed: 15541358]

13. Shah RB, et al. Androgen-independent prostate cancer is a heterogeneous group of diseases: lessons from a rapid autopsy program. Cancer Res 2004;64:9209-9216. [PubMed: 15604294]

14. Rinker-Schaeffer CW, et al. Metastasis suppressor proteins: discovery, molecular mechanisms, and clinical application. Clin Cancer Res 2006;12:3882-3889. [PubMed: 16818682]

15. Nash KT, et al. Requirement of KISS1 secretion for multiple organ metastasis suppression and maintenance of tumor dormancy. J Natl Cancer Inst 2007;99:309-321. [PubMed: 17312308]

16. Chekmareva M, et al. Chromosome 17-mediated dormancy of AT6.1 prostate cancer micrometastases. Cancer Res 1998;58:4963-4969. [PubMed: 9810006]

17. Nagle JA, et al. Involvement of insulin receptor substrate 2 in mammary tumor metastasis. Mol Cell Biol 2004;24:9726-9735. [PubMed: 15509777]

18. Martin SS, et al. A cytoskeleton-based functional genetic screen identifies Bcl-xL as an enhancer of metastasis, but not primary tumor growth. Oncogene 2004;23:4641-4645. [PubMed: 15064711]

19. Criscuoli ML, et al. Tumor metastasis but not tumor growth is dependent on Src-mediated vascular permeability. Blood 2005;105:1508-1514. [PubMed: 15486073]

20. Witz IP, Levy-Nissenbaum O. The tumor microenvironment in the post-PAGET era. Cancer Lett 2006;242:1-10. [PubMed: 16413116]

21. Postovit LM, et al. Influence of the microenvironment on melanoma cell fate determination and phenotype. Cancer Res 2006;66:7833-7836. [PubMed: 16912153] 
22. Al-Mehdi A, et al. Intravascular origin of metastasis from the proliferation of endothelium-attached tumor cells: a new model for metastasis. Nat Med 2000;6:100-102. [PubMed: 10613833]

23. Kaplan RN, et al. VEGFR1-positive haematopoietic bone marrow progenitors initiate the premetastatic niche. Nature 2005;438:820-827. [PubMed: 16341007]

24. VanderGriend D, et al. Suppression of metastatic colonization by the context-dependent activation of the c-jun NH2-terminal kinase kinases JNKK1/MKK4 and MKK7. Cancer Res 2005;65:1098410991. [PubMed: 16322247]

25. Hicklin DJ, Ellis LM. Role of the vascular endothelial growth factor pathway in tumor growth and angiogenesis. J Clin Oncol 2005;23:1011-1027. [PubMed: 15585754]

26. Hurwitz H, et al. Bevacizumab plus irinotecan, fluorouracil, and leucovorin for metastatic colorectal cancer. N Engl J Med 2004;350:2335-2342. [PubMed: 15175435]

27. Yang JC, et al. A randomized trial of bevacizumab, an anti-vascular endothelial growth factor antibody, for metastatic renal cancer. N Engl J Med 2003;349:427-434. [PubMed: 12890841]

28. Van den Eynden GG, et al. Differential expression of hypoxia and (lymph)angiogenesis-related genes at different metastatic sites in breast cancer. Clin Exp Metastasis 2007;24:13-23. [PubMed: 17295094]

29. Casanovas O, et al. Drug resistance by evasion of antiangiogenic targeting of VEGF signaling in latestage pancreatic islet tumors. Cancer Cell 2005;8:299-309. [PubMed: 16226705]

30. Dorrell MI, et al. Combination angiostatic therapy completely inhibits ocular and tumor angiogenesis. Proc Natl Acad Sci USA 2007;104:967-972. [PubMed: 17210921]

31. Wang J, et al. A glycolytic mechanism regulating an angiogenic switch in prostate cancer. Cancer Res 2007;67:149-159. [PubMed: 17210694]

32. Stessels F, et al. Breast adenocarcinoma liver metastases, in contrast to colorectal cancer liver metastases, display a non-angiogenic growth pattern that preserves the stroma and lacks hypoxia. $\mathrm{Br}$ J Cancer 2004;90:1429-1436. [PubMed: 15054467]

33. Kusters B, et al. Vascular endothelial growth factor-A(165) induces progression of melanoma brain metastases without induction of sprouting angiogenesis. Cancer Res 2002;62:341-345. [PubMed: 11809675]

34. Noguera-Troise I, et al. Blockade of Dll4 inhibits tumour growth by promoting non-productive angiogenesis. Nature 2006;444:1032-1037. [PubMed: 17183313]

35. Ridgway J, et al. Inhibition of Dll4 signalling inhibits tumour growth by deregulating angiogenesis. Nature 2006;444:1083-1087. [PubMed: 17183323]

36. MacKie RM, et al. Fatal melanoma transferred in a donated kidney 16 years after melanoma surgery. N Engl J Med 2003;348:567-568. [PubMed: 12571271]

37. Naumov G, et al. Persistence of solitary mammary carcinoma cells in a secondary site: a possible contributor to dormancy. Cancer Res 2002;62:2162-2168. [PubMed: 11929839]

38. Holmgren L, et al. Dormancy of micrometastases: balanced proliferation and apoptosis in the presence of angiogenesis suppression. Nat Med 1995;1:149-153. [PubMed: 7585012]

39. Nierodzik ML, Karpatkin S. Thrombin induces tumor growth, metastasis, and angiogenesis: evidence for a thrombin-regulated dormant tumor phenotype. Cancer Cell 2006;10:355-362. [PubMed: 17097558]

40. Dalton W. The tumor microenvironment as a determinant of drug response and resistance. Drug Resist Updat 1999;2:285-288. [PubMed: 11504502]

41. Neville-Webbe HL, et al. Osteoprotegerin (OPG) produced by bone marrow stromal cells protects breast cancer cells from TRAIL-induced apoptosis. Breast Cancer Res Treat 2004;86:269-279. [PubMed: 15567943]

42. Efstathiou $\mathrm{E}$, et al. Initial modulation of the tumor microenvironment accounts for thalidomide activity in prostate cancer. Clin Cancer Res 2007;13:1224-1231. [PubMed: 17317833]

43. Bird NC, et al. Biology of colorectal liver metastases: a review. J Surg Oncol 2006;94:68-80. [PubMed: 16788948]

44. Deeken JF, Loscher W. The blood-brain barrier and cancer: transporters, treatment, and trojan horses. Clin Cancer Res 2007;13:1663-1674. [PubMed: 17363519] 
45. Palmieri D, et al. The biology of metastasis to a sanctuary site. Clin Cancer Res 2007;13:1656-1662. [PubMed: 17363518]

46. Roodman G. Mechanisms of bone metastasis. N Engl J Med 2004;350:1655-1664. [PubMed: 15084698]

47. Kang Y, et al. A multigenic program mediating breast cancer metastasis to bone. Cancer Cell 2003;3:537-549. [PubMed: 12842083]

48. Roudier MP, et al. Phenotypic heterogeneity of end-stage prostate carcinoma metastatic to bone. Hum Pathol 2003;34:646-653. [PubMed: 12874759]

49. Mundy G. Metastasis to the bone: causes, consequences and therapeutic opportunities. Nat Rev Cancer 2002;2:584-593. [PubMed: 12154351]

50. Kishida Y, et al. Parthenolide, a natural inhibitor of nuclear factor-kappaB, inhibits lung colonization of murine osteosarcoma cells. Clin Cancer Res 2007;13:59-67. [PubMed: 17200339]

51. Shannon KE, et al. Anti-metastatic properties of RGD-peptidomimetic agents S137 and S247. Clin Exp Metastasis 2004;21:129-138. [PubMed: 15168730]

52. Khalili P, et al. Effect of Herceptin on the development and progression of skeletal metastases in a xenograft model of human breast cancer. Oncogene 2005;24:6657-6666. [PubMed: 16091754]

53. Slamon $\mathrm{D}$, et al. Use of chemotherapy plus a monoclonal antibody against HER 2 for metastatic breast cancer that overexpresses HER2. N Engl J Med 2001;344:783-792. [PubMed: 11248153]

54. Piccart-Gebhart MJ, et al. Trastuzumab after adjuvant chemotherapy in HER2-positive breast cancer. N Engl J Med 2005;353:1659-1672. [PubMed: 16236737]

55. Romond EH, et al. Trastuzumab plus adjuvant chemotherapy for operable HER2-positive breast cancer. N Engl J Med 2005;353:1673-1684. [PubMed: 16236738]

56. Tosi P, et al. First-line therapy with thalidomide, dexamethasone and zoledronic acid decreases bone resorption markers in patients with multiple myeloma. Eur J Haematol 2006;76:399-404. [PubMed: 16480429]

57. Body J, et al. A phase I study of AMGN-007, a recombinant osterogrotegerin construct, in patients with multiple myeloma or breast carcinoma related bone metastases. Cancer 2002;97(3 Suppl):887892. [PubMed: 12548591]

58. Body JJ, et al. A study of the biological receptor activator of nuclear factor-kappaB ligand inhibitor, denosumab, in patients with multiple myeloma or bone metastases from breast cancer. Clin Cancer Res 2006;12:1221-1228. [PubMed: 16489077]

59. Bochner $\mathrm{BH}$, et al. Postoperative nomogram predicting risk of recurrence after radical cystectomy for bladder cancer. J Clin Oncol 2006;24:3967-3972. [PubMed: 16864855]

60. Wedam S, et al. Antiangiogenic and antitumor effects of bevacizumab in patients with inflammatory and locally advanced breast cancer. J Clin Oncol 2006;24:769-777. [PubMed: 16391297]

61. Jubb AM, et al. Impact of vascular endothelial growth factor-A expression, thrombospondin-2 expression, and microvessel density on the treatment effect of bevacizumab in metastatic colorectal cancer. J Clin Oncol 2006;24:217-227. [PubMed: 16365183]

62. Lise M, et al. Colorectal liver metastasis: towards the integration of conventional and molecularly targeted therapeutic approaches. Front Biosci 2005;10:3042-3057. [PubMed: 15970559]

63. Herbst RS, et al. Phase I/II trial evaluating the anti-vascular endothelial growth factor monoclonal antibody bevacizumab in combination with the HER-1/ epidermal growth factor receptor tyrosine kinase inhibitor erlotinib for patients with recurrent non-small-cell lung cancer. J Clin Oncol 2005;23:2544-2555. [PubMed: 15753462]

64. Hainsworth JD, et al. Treatment of metastatic renal cell carcinoma with a combination of bevacizumab and erlotinib. J Clin Oncol 2005;23:7889-7896. [PubMed: 16204015]

65. Miller KD, et al. A multicenter phase II trial of ZD6474, a vascular endothelial growth factor receptor-2 and epidermal growth factor receptor tyrosine kinase inhibitor, in patients with previously treated metastatic breast cancer. Clin Cancer Res 2005;11:3369-3376. [PubMed: 15867237]

66. Paterson AH. The role of bisphosphonates in early breast cancer. Oncologist 2006;11(Suppl 1):S13S19.

67. Diel, I., et al. Monoclonal antibodies to detect breast cancer cells in bone marrow. In: De Vita, VT., et al., editors. Important Advances in Oncology. Philadelphia: Lippincott; 1994. p. 143-164. 
68. Saarto T, et al. Adjuvant clodronate treatment does not reduce the frequency of skeletal metastases in node-positive breast cancer patients; 5-year results of a randomized controlled trial. J Clin Oncol 2001;19:10-17. [PubMed: 11134190]

69. Powles T, et al. Randomized, placebo-controlled trial of clodronate in patients with primary operable breast cancer. J Clin Oncol 2002;20:3219-3224. [PubMed: 12149294]

70. Saarto T, et al. Ten-year follow-up of a randomized controlled trial of adjuvant clodronate treatment in node-positive breast cancer patients. Acta Oncol 2004;43:650-656. [PubMed: 15545185]

71. Bertelli G, et al. Weekly docetaxel and zoledronic acid every 4 weeks in hormone-refractory prostate cancer patients. Cancer Chemother Pharmacol 2006;57:46-51. [PubMed: 16001175]

72. Nelson JB, et al. New bone formation in an osteoblastic tumor model is increased by endothelin-1 overexpression and decreased by endothelin A receptor blockade. Urology 1999;53:1063-1069. [PubMed: 10223507]

73. Carducci MA, Jimeno A. Targeting bone metastasis in prostate cancer with endothelin receptor antagonists. Clin Cancer Res 2006;12:S6296-S6300.

74. Nelson JB, et al. Suppression of prostate cancer induced bone remodeling by the endothelin receptor A antagonist atrasentan. J Urol 2003;169:1143-1149. [PubMed: 12576870]

75. Body JJ, et al. A phase I study of AMGN-0007, a recombinant osteoprotegerin construct, in patients with multiple myeloma or breast carcinoma related bone metastases. Cancer 2003;97:887-892. [PubMed: 12548591]

76. Body JJ, et al. A study of the biological receptor activator of nuclear factor-kappaB ligand inhibitor, denosumab, in patients with multiple myeloma or bone metastases from breast cancer. Clin Cancer Res 2006;12:1221-1228. [PubMed: 16489077]

77. Palmieri D, et al. Medroxyprogesterone acetate elevation of Nm23-H1 metastasis suppressor expression in hormone receptor-negative breast cancer. J Natl Cancer Inst 2005;97:632-642. [PubMed: 15870434]

78. Titus B, et al. Endothelin axis is a target of the lung metastasis suppressor gene RhoGDI2. Cancer Res 2005;65:7320-7327. [PubMed: 16103083]

79. Horak C, et al. Nm23-H1 suppresses metastasis by inhibiting expression of the lysophosphatidic acid receptor EDG2. Cancer Res 2007;67:11751-11759. [PubMed: 18089805]

80. Orsini MJ, et al. Metastin (KiSS-1) mimetics identified from peptide structure-activity relationshipderived pharmacophores and directed small molecule database screening. J Med Chem 2007;50:462471. [PubMed: 17266198]

81. Gupta GP, et al. Mediators of vascular remodelling co-opted for sequential steps in lung metastasis. Nature 2007;446:765-770. [PubMed: 17429393]

82. Kang Y, et al. Breast cancer bone metastasis mediated by the Smad tumor suppressor pathway. Proc Natl Acad Sci USA 2005;102:13909-13914. [PubMed: 16172383]

83. Nam JS, et al. Bone sialoprotein mediates the tumor cell-targeted prometastatic activity of transforming growth factor beta in a mouse model of breast cancer. Cancer Res 2006;66:6327-6335. [PubMed: 16778210]

84. Zhang Q, et al. Blockade of transforming growth factor- $\beta$ signaling in tumor-reactive CD8(+) T cells activates the antitumor immune response cycle. Mol Cancer Ther 2006;5:1733-1743. [PubMed: 16891459]

85. Biswas $\mathrm{S}$, et al. Inhibition of TGF-beta with neutralizing antibodies prevents radiation-induced acceleration of metastatic cancer progression. J Clin Invest 2007;117:1305-1313. [PubMed: 17415413]

86. Biswas $\mathrm{S}$, et al. Inhibition of transforming growth factor-beta signaling in human cancer: targeting a tumor suppressor network as a therapeutic strategy. Clin Cancer Res 2006;12:4142-4146. [PubMed: 16857784]

87. Ono K, et al. Involvement of hepatocyte growth factor in the development of bone metastasis of a mouse mammary cancer cell line, BALB/c-MC. Bone 2006;39:27-34. [PubMed: 16459153]

88. Peruzzi B, Bottaro DP. Targeting the c-Met signaling pathway in cancer. Clin Cancer Res 2006;12:3657-3660. [PubMed: 16778093] 
89. Rucci N, et al. Inhibition of protein kinase c-Src reduces the incidence of breast cancer metastases and increases survival in mice: implications for therapy. J Pharmacol Exp Ther 2006;318:161-172. [PubMed: 16627750]

90. Jallal H, et al. A Src/Abl kinase inhibitor, SKI-606, blocks breast cancer invasion, growth, and metastasis in vitro and in vivo. Cancer Res 2007;67:1580-1588. [PubMed: 17308097]

91. Al-Hajj M, et al. Prospective identification of tumorigenic breast cancer cells. Proc Natl Acad Sci USA 2003;100:3983-3988. [PubMed: 12629218]

92. Jones DH, et al. Regulation of cancer cell migration and bone metastasis by RANKL. Nature 2006;440:692-696. [PubMed: 16572175]

93. Park BK, et al. NF-kappaB in breast cancer cells promotes osteolytic bone metastasis by inducing osteoclastogenesis via GM-CSF. Nat Med 2007;13:62-69. [PubMed: 17159986]

94. Horsman MR, Siemann DW. Pathophysiologic effects of vascular-targeting agents and the implications for combination with conventional therapies. Cancer Res 2006;66:11520-11539. [PubMed: 17178843]

95. Bandyopadhyay A, et al. Inhibition of pulmonary and skeletal metastasis by a transforming growth factor-beta type I receptor kinase inhibitor. Cancer Res 2006;66:6714-6721. [PubMed: 16818646]

96. Lang JY, et al. Antimetastatic effect of salvicine on human breast cancer MDA-MB-435 orthotopic xenograft is closely related to Rho-dependent pathway. Clin Cancer Res 2005;11:3455-3464. [PubMed: 15867248]

97. Palm D, et al. The norepinephrine-driven metastasis development of PC-3 human prostate cancer cells in BALB/c nude mice is inhibited by beta-blockers. Int J Cancer 2006;118:2744-2749. [PubMed: 16381019]

98. Giubellino A, et al. Inhibition of tumor metastasis by a growth factor receptor bound protein $2 \mathrm{Src}$ homology 2 domain-binding antagonist. Cancer Res 2007;67:6012-6016. [PubMed: 17616655]

99. Cairns RA, Hill RP. Acute hypoxia enhances spontaneous lymph node metastasis in an orthotopic murine model of human cervical carcinoma. Cancer Res 2004;64:2054-2061. [PubMed: 15026343]

100. Cassinelli G, et al. Inhibition of c-Met and prevention of spontaneous metastatic spreading by the 2-indolinone RPI-1. Mol Cancer Ther 2006;5:2388-2397. [PubMed: 16985073]

\section{Acknowledgements}

PS Steeg was funded by the Intramural research program of the Center for Cancer Research, NCI, and D Theodorescu was supported by NIH grants CA104106 and CA075115. 


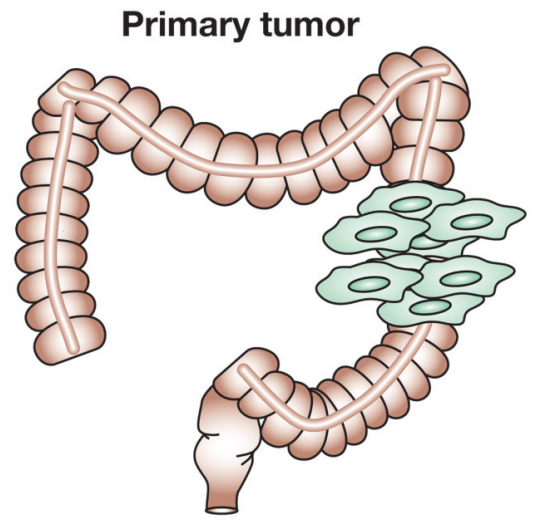

CGH

Gain of $7,3 q, 13 q, 20, x$

Loss of $4,8 p, 17 p, 18$

Ras mutation

$16 / 30$ wild type

$14 / 30$ mutant

RNA

Greater E-cadherin, Fas

Lower DPD, TP levels

IHC

Greater E-cadherin, CD44, EphB staining

More MMP-9+ macrophages at tumor border

\section{Liver metastasis}

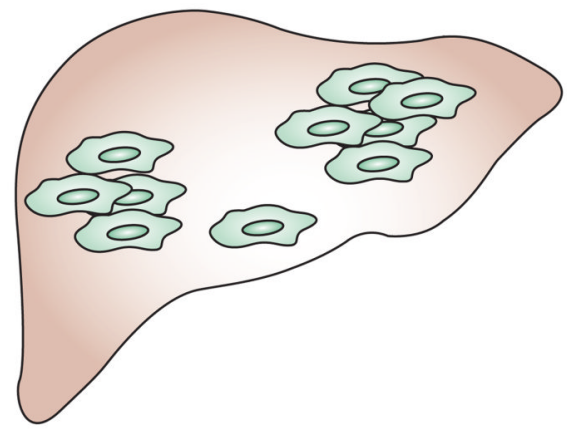

\section{CGH}

Additional gain of 1q, 6, 8q, 11, 12, 16, 17q, 19

Additional loss of 1p, 6q, 9p, 10q, 14q, 15q

Ras mutation

4/16 metastases of these tumors were mutant $5 / 14$ metastases of these tumors were wild type

\section{RNA}

Greater GPX3, MAP2, N-Wasp, NCAML1, $\mathrm{T} \beta 4, \beta$-catenin, c-Myc, MMP-7

\section{Figure 1.}

Molecular distinctions between primary colorectal carcinomas and their liver metastases. While primary tumors and metastases are identical in many respects, differences exist. Two types of comparisons are listed: molecular analyses of matched primary tumors and resected liver metastases (mutation, RNA and immunohistochemistry data), and a meta-analysis of CGH data generated from both matched and unmatched samples. References are listed in Supplementary Table 1. Abbreviations: CGH, comparative genomic hybridization; IHC, immunohistochemistry. 
Metastatic colonization

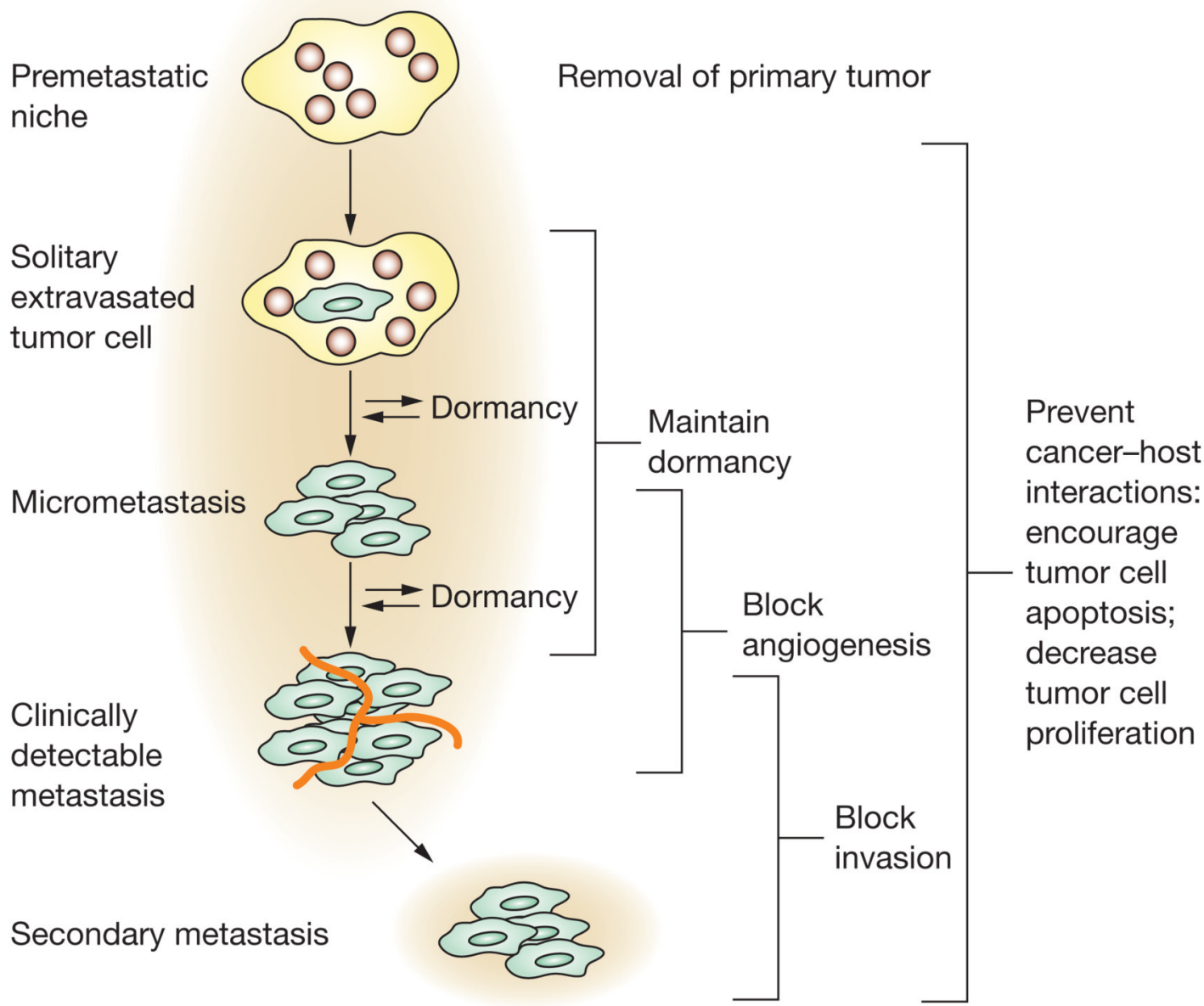

Figure 2.

Metastatic colonization. Metastatic colonization represents a prime window of opportunity to interrupt the metastatic process. Growth in a distant site has similarities and differences to that in the primary tumor site. Steps in metastatic colonization are listed on the left, with potential breaks for dormancy shown. Potential therapeutic strategies are listed to the right. 


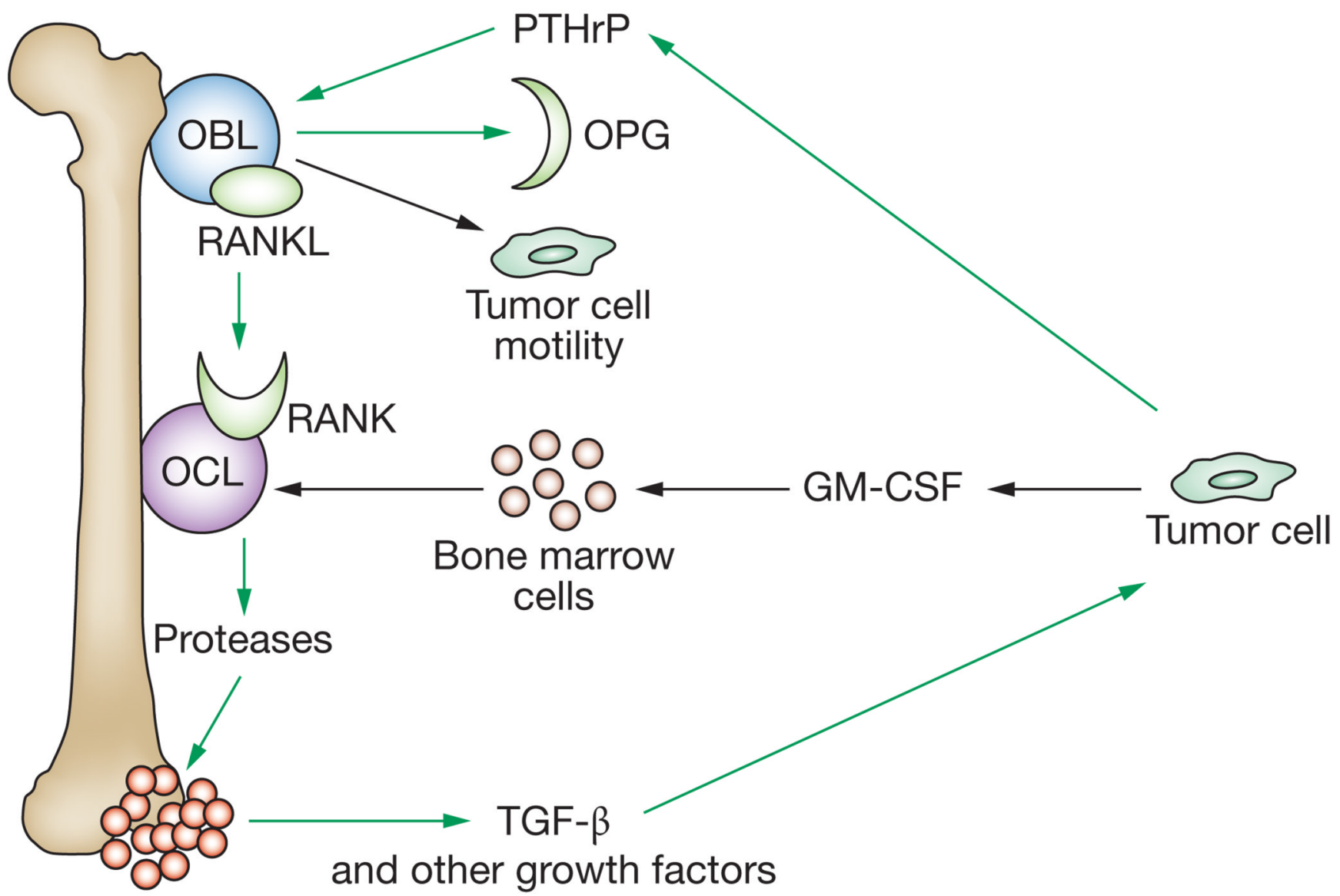

Figure 3.

The bone metastasis 'vicious' cycle with recent updates. Metastatic tumor cells interact with the bone microenvironment to facilitate osteolytic colonization. Tumor cells secrete PTHrP, which stimulates osteoblasts (bone-forming cells) to produce both a membrane-bound RANKL and OPG, a soluble decoy receptor for RANKL. It is the ratio of RANKL to OPG that determines osteoclast (bone-degrading cell) activation, through its receptor for RANKL. Activated osteoclasts degrade the bone matrix, releasing into the local microenvironment embedded growth factors including TGF- $\beta$. TGF- $\beta$ stimulates tumor-cell PTHrP production, renewing the cycle. Recently, RANKL was reported to stimulate tumor cells as well as osteoclasts, inducing motility that could spread bone colonization. ${ }^{92}$ Tumor cells also produce GM-CSF, a hematopoietic growth factor used in cancer therapy. GM-CSF, in turn, stimulated bone marrow cells to produce more osteoclasts, amplifying the cycle. ${ }^{93}$ Abbreviations: GMCSF, granulocyte macrophage-colony stimulating factor; OBL, osteoblast-like cells; OCL, osteoclast-like cells; OPG, osteoprotegerin; PTHrP, parathyroid-hormone-related protein; RANKL, receptor activator of NF- $\kappa$ B ligand; TGF- $\beta$, transforming growth factor- $\beta$. 


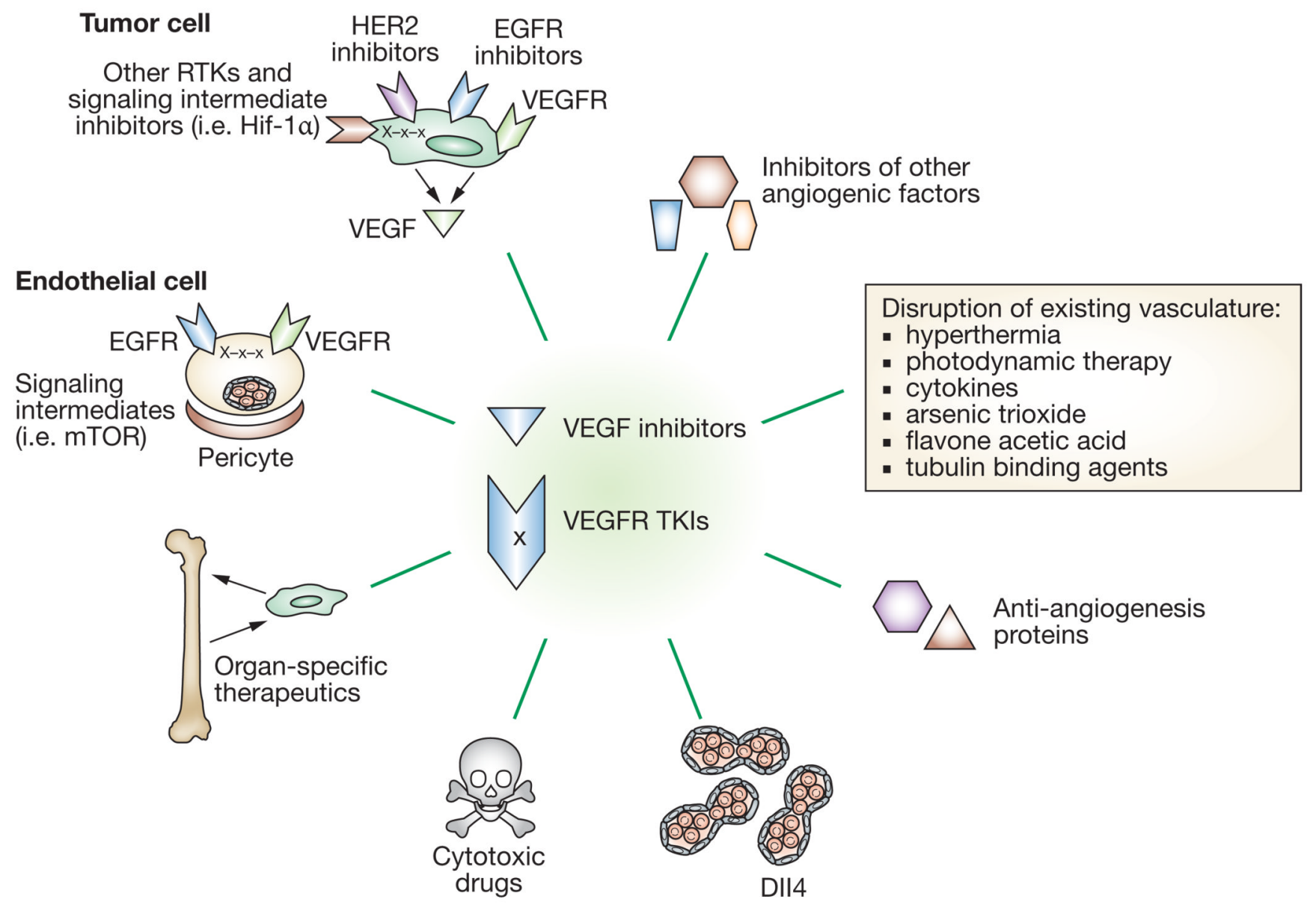

Figure 4.

An overwhelming number of potential rational combinations of drugs are available for metastatic colonization: angiogenesis as an example. Inhibitors of VEGF and its receptor (VEGFR) have been brought to clinical testing. Potential rational combinations are shown by lines, including aspects of tumor cell biology, the microenvironment and traditional cytotoxics. 25,34,35,94 Abbreviations: EGFR, epidermal growth factor receptor; HER2, human epidermal growth factor receptor 2; RTKs, receptor tyrosine kinases; TKIs, tyrosine kinase inhibitors; VEGF, vascular endothelial growth factor. 
Table 1

In model systems, therapeutics and environmental conditions can differentially affect primary tumors and metastases.

\begin{tabular}{|c|c|c|}
\hline Histology & Findings & Reference \\
\hline \multirow[t]{3}{*}{ Breast } & $\begin{array}{l}\text { Systemic administration of a TGF- } \beta \text { RI-I inhibitor reduced the number of lung } \\
\text { metastases and the incidence and burden of bone metastases with no effect } \\
\text { on the orthotopic primary tumor }\end{array}$ & $\begin{array}{l}\text { Bandyopadhyay et al. } \\
(2006)^{95}\end{array}$ \\
\hline & $\begin{array}{l}\text { Integrin-based peptidomimetics reduced lung metastasis by } 44-68 \% \text {, with } \\
\text { no effect on primary tumor growth and only a minor difference in the number } \\
\text { of circulating tumor cells }\end{array}$ & Shannon et al. (2004) 51 \\
\hline & $\begin{array}{l}\text { The topoisomerase II inhibitor salvicine reduced lung metastasis by } 33-68 \% \text {, } \\
\text { with no diminution of primary tumor growth, via a RhoC pathway }\end{array}$ & Lang et al. (2005) 96 \\
\hline Osteosarcoma & $\begin{array}{l}\text { Parthenolide, a NF- } \kappa \mathrm{B} \text { directed agent, inhibited lung metastasis fourfold in } \\
\text { a prevention model with no significant effect on the growth of primary } \\
\text { tumors. A similar trend was reported for mutant I } \mathrm{B} \alpha \text { expression }\end{array}$ & Kishida et al. $(2007)^{50}$ \\
\hline Prostate & $\begin{array}{l}\text { Norepinephrine increased, and propranolol decreased the lumbar lymph- } \\
\text { node metastases of PC-3 cancer cells; growth of the primary tumor was not } \\
\text { affected }\end{array}$ & Palm et al. (2006) $)^{97}$ \\
\hline Prostate and melanoma & $\begin{array}{l}\text { PC } 3 \mathrm{M} \text { prostate and B16 melanoma cells were treated ex vivo with C90, a } \\
\text { Grb-2 inhibitor, and injected. The compound inhibited lung metastasis by } \\
50 \% \text {, with no significant effect on primary tumor size }\end{array}$ & Giubellino et al. (200798 \\
\hline Cervical & $\begin{array}{l}\text { Acute hypoxia significantly decreased primary tumor size but increased the } \\
\text { number of positive lymph nodes and the size of the lesions }\end{array}$ & Cairns and Hill (2004) 99 \\
\hline Pancreatic & $\begin{array}{l}\text { Cyclopamine reduced the incidence of distant metastases to the spleen, liver, } \\
\text { lymph nodes and peritoneum, with no significant effect on primary tumor } \\
\text { volume }\end{array}$ & Feldmann et al. $(2007)^{9}$ \\
\hline Lung & $\begin{array}{l}\text { RPI-1, a c-Met inhibitor, did not inhibit subcutaneous tumors produced by } \\
\text { H460 lung cancer cells at } 100 \mathrm{mg} / \mathrm{kg} \text {, but reduced metastases by } 57 \%\end{array}$ & Cassinelli et al. (2006) ${ }^{100}$ \\
\hline
\end{tabular}

